\title{
Stereotactic surgery for neurocysticercosis of the 4th ventricle: illustrative cases
}

\author{
Luis J. Saavedra, MD, ${ }^{1}$ Carlos M. Vásquez, MD, ${ }^{1}$ Hector H. García, MD, PhD, ${ }^{2}$ Luis A. Antonio, MD, ${ }^{1}$ Yelimer Caucha, MD, ${ }^{1}$ \\ Jesús Félix, MD, ${ }^{1}$ Jorge $\mathrm{E}$. Medina, MD, ${ }^{1}$ and William W. Lines, $\mathrm{MD}^{1}$
}

${ }^{1}$ Departamento de Investigación, Docencia y Atención Especializada en Neurocirugía and ${ }^{2}$ Cysticercosis Unit, Instituto Nacional de Ciencias Neurológicas, Lima, Perú

BACKGROUND Neurocysticercosis, caused by the larval stage of Taenia solium, affects the cerebral ventricles in 20-30\% of cases and may lead to hydrocephalus and other neurological morbidity. Conventional treatment for cysts in the 4th ventricle includes open surgery (suboccipital approach) and neuroendoscopy, with the latter being the option of choice. Stereotactic surgery, minimally invasive, offers a good alternative for this type of deep lesion.

OBSERVATIONS The authors report the cases of two women, 30 and 45 years old, who presented with headache, dizziness, and ataxia and were diagnosed with 4th ventricle cysticercosis. Magnetic resonance imaging (MRI) revealed dilated 4th ventricles (approximately $2.5 \mathrm{~cm}$ in both cases, with cystic images inside the ventricular cavity). Both patients were treated with stereotactic surgery via a suboccipital transcerebellar approach. Cyst material was extracted, and the diagnosis was confirmed by pathological examination. The surgeries had no complications and resulted in clinical improvement. Control MRI scans showed reduction of the volume of the ventricle without residual cysts.

LESSONS Minimally invasive stereotactic surgery provided a safe alternative for 4th ventricle neurocysticercosis cysts, with more benefits than risks in comparison with conventional techniques.

https://thejns.org/doi/abs/10.3171/CASE21279

KEYWORDS neurocysticercosis; stereotactic surgery; 4th ventricle

Neurocysticercosis (NCC), a zoonosis caused by the invasion of the human central nervous system by the cystic larval stage of the pork tapeworm (Taenia solium), is the most frequent neurological helminth infection worldwide and one of the most frequent causes of epilepsy and hydrocephalus in adult patients. ${ }^{1-4}$ NCC can be parenchymal, ventricular, or subarachnoid. Ventricular NCC can be life threatening due to the risk of obstructive hydrocephalus, either because of the cyst or by induced ependymitis, hence the importance of prompt diagnosis and treatment. ${ }^{5,6}$ Ventricular NCC occurs in $20-30 \%$ of all NCC patients. Cysts may be adhered to the ependyma or free inside the ventricular cavity. ${ }^{7,8}$ Neuroendoscopic exeresis is the treatment option recommended by current management guidelines, with subsequent medical treatment with albendazole if there is reason to believe that viable cysts remain after surgery. ${ }^{2,4,9}$ To the best of our knowledge, there are no published reports of stereotactic surgery for 4 th ventricular NCC. We report two patients with 4 th ventricular NCC who were successfully treated with stereotactic surgery.

\section{Illustrative Cases}

\section{Case 1}

A 30-year-old woman with a 1-year history of hydrocephalus treated with peritoneal ventricular shunt consulted for headache, nausea, vomiting, dizziness, and progressive walking instability of 2 months' evolution. Neurological examination revealed global disorientation and cerebellar ataxia without other neurological signs. Magnetic resonance imaging (MRI) of the brain (Fig. 1) in fast imaging employing steady-state acquisition (FIESTA), fluid-attenuated inversion recovery (FLAIR), and T1-weighted sequences before and after gadolinium injection revealed multiple cystic lesions in the 4th ventricle, associated with an increase in size $(2.6 \mathrm{~cm}$ in anteroposterior diameter). Immunodiagnosis was performed using enzyme-linked immunoelectrotransfer blot (EITB) analysis (Western blot; sensitivity of $98 \%$ and specificity of $100 \%$ in patients with two or more viable brain lesions), which showed reactivity to all seven diagnostic antibody bands (Table 1). Blood

ABBREVIATIONS CSF = cerebrospinal fluid; EITB = enzyme-linked immunoelectrotransfer blot; FIESTA = fast imaging employing steady-state acquisition; FLAIR = fluid-attenuated inversion recovery; MRI = magnetic resonance imaging; NCC = neurocysticercosis.

INCLUDE WHEN CITING Published July 19, 2021; DOI: 10.3171/CASE21279.

SUBMITTED May 11, 2021. ACCEPTED May 28, 2021.

(C) 2021 The authors, CC BY-NC-ND 4.0 (http://creativecommons.org/licenses/by-nc-nd/4.0/). 

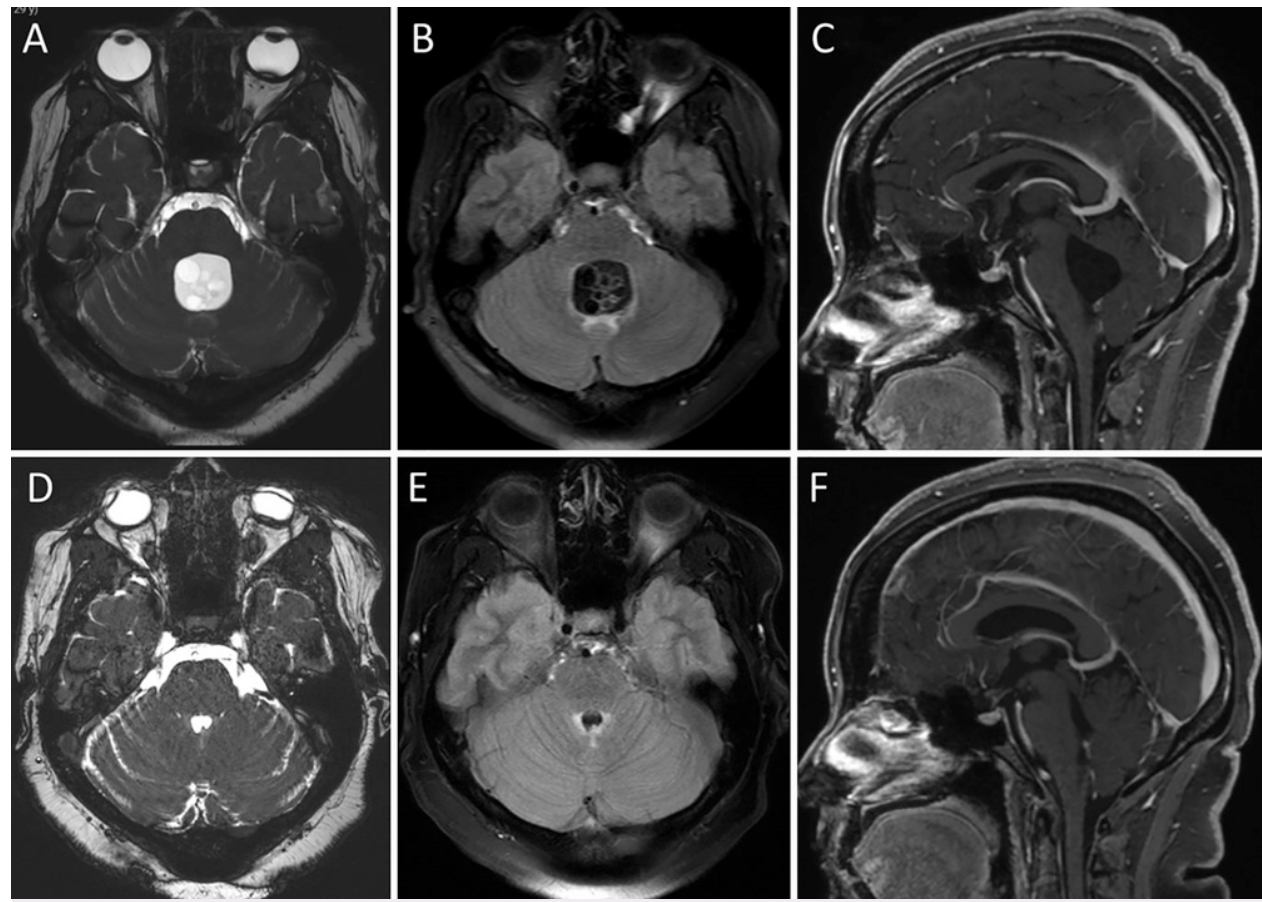

FIG. 1. MRI studies of case 1. A-C: Preoperative imaging. Dilated 4th ventricle with images suggestive of cystic lesions in its interior. D-F: Control MRI at 3 months after surgery. The diameter of the 4th ventricle has decreased, and there are no remnant cysts ( $\mathbf{A}$ and $\mathbf{D}$, axial FIESTA; $\mathbf{B}$ and $\mathbf{E}$, axial FLAIR; $\mathbf{C}$ and $\mathbf{F}$, sagittal postcontrast T1).

counts and biochemistry values were within normal parameters. The patient's treatment was planned for cyst exeresis by stereotactic surgery. (Details are described below under Surgery.)

There were no complications, and she was discharged on day 4 after surgery without any neurological deficit. Pathology confirmed the diagnosis (Fig. 2). Two months after surgery, she received antiparasitic treatment (albendazole $400 \mathrm{mg} /$ day for 30 days, with concomitant dexamethasone at $8 \mathrm{mg} /$ day for 2 weeks and then gradually withdrawn for 6 weeks). At 3 and 24 months, control brain MRI was performed, which showed that the 4th ventricle had recovered its size and that there were no remnant cystic lesions inside. At her 2-year follow-up visit, she was asymptomatic (modified Rankin scale score 0 ).

\section{Case 2}

A 45-year-old woman with no past medical history consulted for a 15month history of progressive headache and dizziness. Her neurological examination revealed disorientation in time and cerebellar ataxia without other neurological findings. Brain MRI (Fig. 3) in FIESTA, FLAIR, and T1-weighted sequences before and after gadolinium injection revealed a cystic lesion inside the 4th ventricle with an apparent scolex, associated with increased ventricular size $(2.5 \mathrm{~cm}$ in anteroposterior diameter). The result of serum EITB for neurocysticercosis was strongly positive (seven bands). The patient's blood counts and biochemistry values were within normal parameters. She was programmed for cyst excision by stereotactic surgery. (Details are described below under Surgery.)

There were no complications, and she was discharged on day 3 after surgery without any neurological deficit. Pathology confirmed the diagnosis (Fig. 2). Two months after surgery, she received antiparasitic treatment as part of a trial comparing albendazole alone with combined albendazole plus praziquantel with concomitant dexamethasone. At 4 and 12 months, control brain MRI was performed, which showed that the 4th ventricle had recovered its size and that there were no remnant cystic lesions inside. In her follow-up visits at 4, 9, and 12 months, she reported mild intermittent headache only (modified Rankin scale score 1).

\section{Surgery}

Individuals with ventricles with a diameter $>2 \mathrm{~cm}$ were considered eligible for this procedure. Local anesthesia plus sedation was

TABLE 1. Epidemiological, clinical, and surgical characteristics of the patients

\begin{tabular}{|c|c|c|c|c|c|c|c|}
\hline Case & Sex, Age (yrs) & Symptoms & Neurological Findings & $\begin{array}{c}\text { Ventricular } \\
\text { Diameter (mm) }\end{array}$ & Anesthesia & $\begin{array}{c}\text { Surgical } \\
\text { Time } \\
\text { (mins) }\end{array}$ & $\begin{array}{l}\text { Modified Rankin } \\
\text { Scale score } \\
\text { at } 12 \text { Mos }\end{array}$ \\
\hline 1 & $\mathrm{~F}, 30$ & $\begin{array}{l}\text { Headache, nausea, vomiting, } \\
\text { dizziness, walking instability }\end{array}$ & $\begin{array}{l}\text { Global disorientation, } \\
\text { cerebellar ataxia }\end{array}$ & $26.6 \times 24.1$ & Local & 95 & 0 \\
\hline 2 & $F, 45$ & Headache, dizziness & $\begin{array}{l}\text { Disorientation in time, } \\
\text { cerebellar ataxia }\end{array}$ & $21.9 \times 25.6$ & Local & 86 & 1 \\
\hline
\end{tabular}



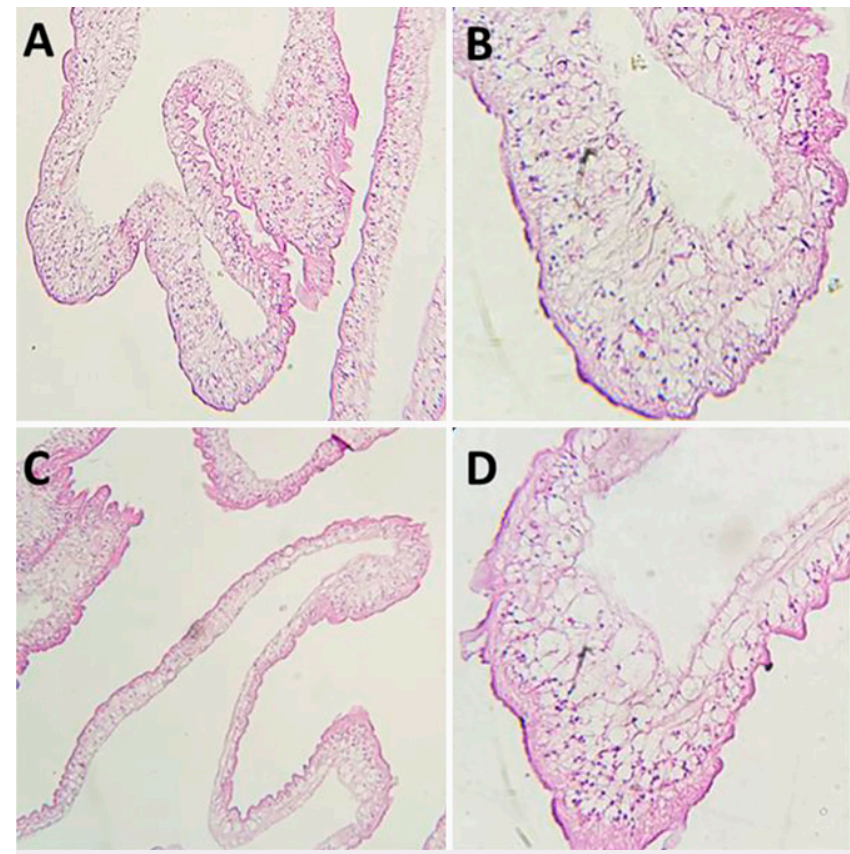

FIG. 2. Cysticercal membranes extracted by stereotaxis. A and B: Case 1. C and D: Case 2. Typical double-layer eosinophilic membranes are shown. Hematoxylin and eosin stain; Original magnifications $\times 10($ A and $\mathbf{C})$ and $\times 40(B$ and $D)$. used in both patients. The Micromar stereotactic frame was used; it has a 2-mm diameter biopsy cannula composed of two hollow cannulas, one inside the other. Both cannulas have a $1-\mathrm{cm}$ rectangular side window located $1 \mathrm{~mm}$ from the lower end. The stereotactic frame was placed at the level of the superior temporal line after infiltration with lidocaine without epinephrine at the points where the pins were fixed. Once the frame was set, contrast-enhanced computed tomography was performed with a standard protocol for stereotaxis, and the target (midpoint of the 4th ventricle) coordinates were calculated (Fig. 4).

We used a transcerebellar suboccipital approach with the patient in the lateral decubitus position. The incision site was marked $3 \mathrm{~cm}$ lateral to the midline and $3 \mathrm{~cm}$ below the projection of the transverse sinus. Once in the bone plane (occipital scale), a trepan, an "X" durotomy, and finally a small corticotomy were performed. The stereotactic frame arch and guide were placed with the previously calculated coordinates, and the closed cannula was introduced (connected to a triple-line system with extension coupled to a $20-\mathrm{ml}$ syringe with saline solution) across the cerebellar hemisphere direct to the 4th ventricle. Once the cannula was set in position, the position was verified by initial aspiration, obtaining cerebrospinal fluid (CSF) in both cases, and then very gentle aspiration was applied until cyst membranes were extracted. If any resistance is noted, the membrane is fragmented with the cutter cannula mechanism, and the process continues until only CSF comes out. No other traction instrumentation (such as forceps) was used. This procedure was repeated at $0^{\circ}, 90^{\circ}, 180^{\circ}$, and $270^{\circ}$ until only CSF was obtained without greater resistance. The cyst membranes were extracted without difficulty in both cases, and there were no complications.
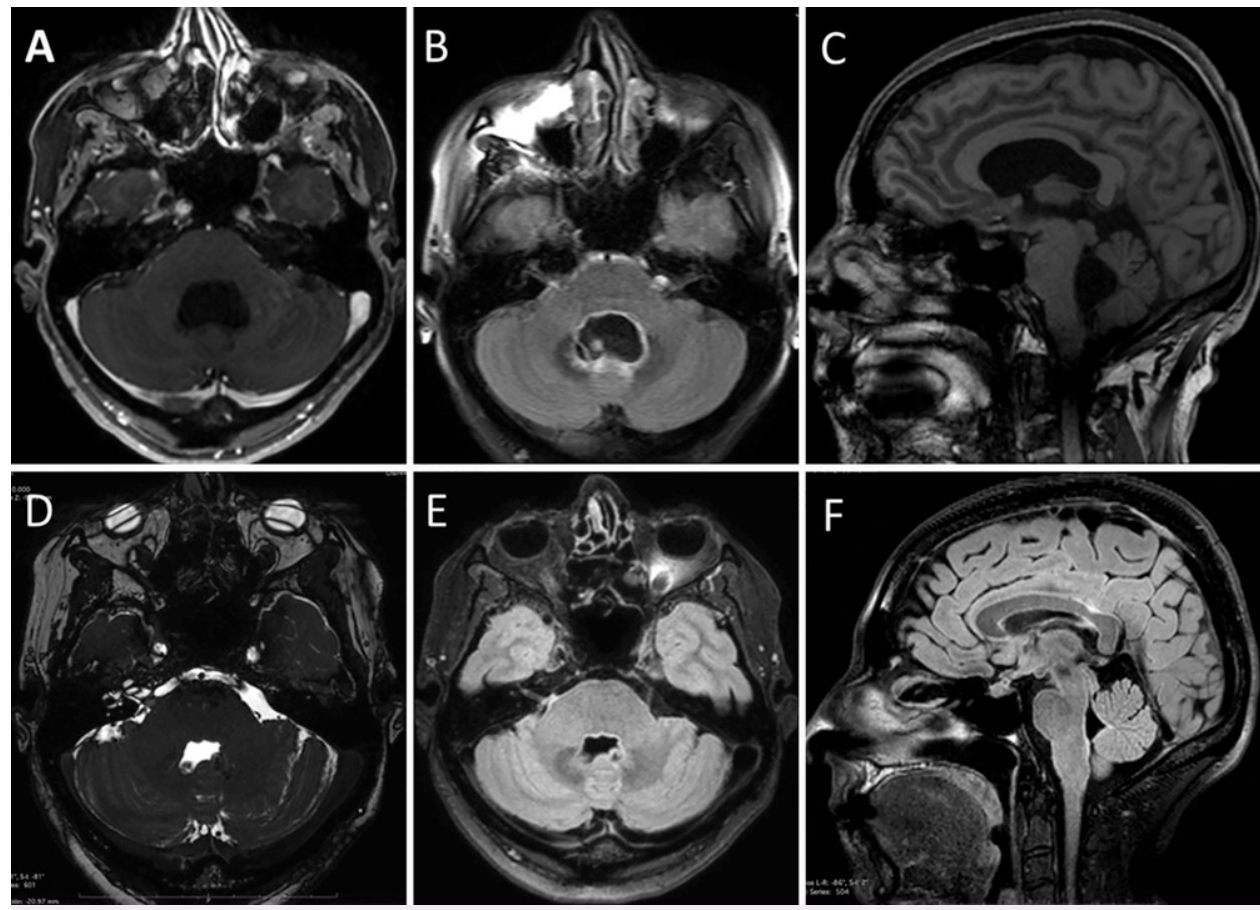

FIG. 3. MRI scans of case 2. A-C: Preoperative imaging. Dilated 4th ventricle with images suggestive of cystic lesions in its interior. D-F: Control MRI at 4 months after surgery. The diameter of the 4 th ventricle has decreased, and there are no remnant cysts (A and $\mathbf{D}$, axial FIESTA; $\mathbf{B}$ and $\mathbf{E}$, axial FLAIR; $\mathbf{C}$ and $\mathbf{F}$, sagittal postcontrast T1). 

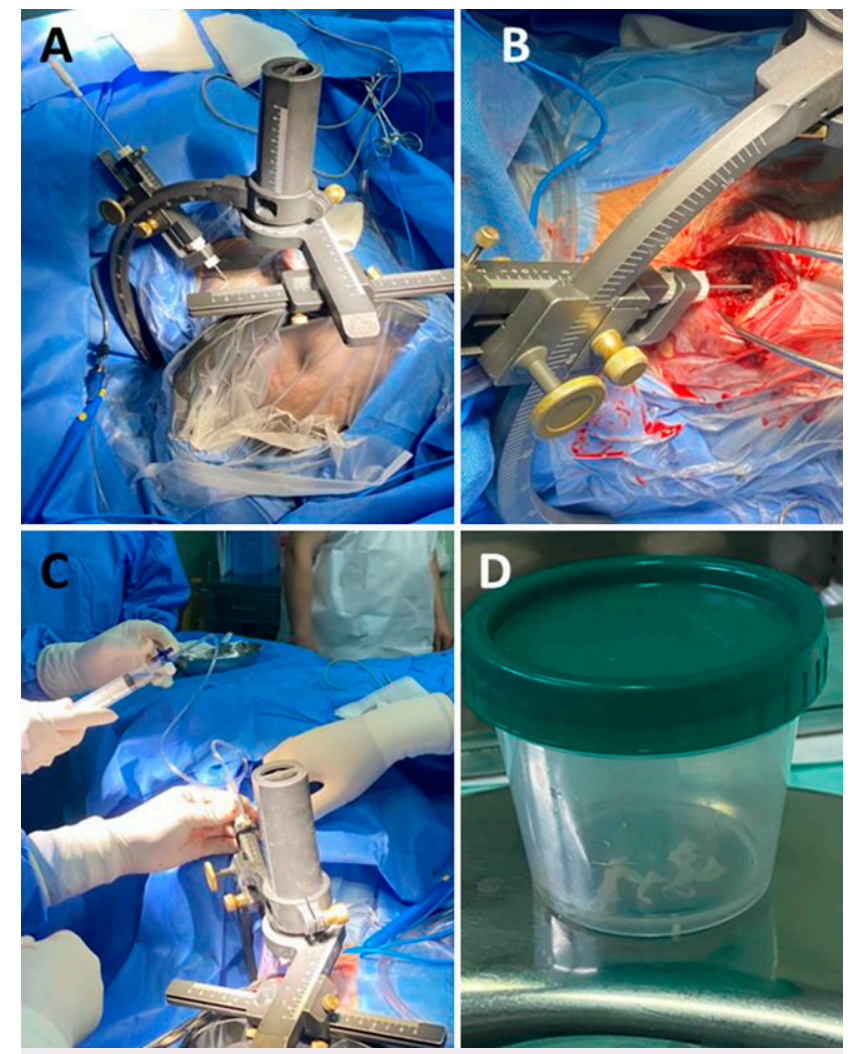

FIG. 4. Photographs of case 2 stereotactic surgery for 4 th ventricular NCC. A: Patient with stereotactic frame in lateral decubitus with cannula at the approach point. B: Suboccipital trepanation with cannula in position, with projection to the 4th ventricle. C: Cannula in final position, and removal of cysts by aspiration. D: Cyst membranes removed.

\section{Discussion}

\section{Observations}

Current neurosurgical approaches in NCC surgery are based on conventional approaches (microsurgical dissection) and neuroendoscopy, which is currently considered the method of choice for ventricular cysts. The main problem in 4th ventricular NCC is the development of acute obstructive hydrocephalus, which can be lethal and must be rapidly approached. Bruns syndrome, described in 1906, occurs due to free cysts in the ventricular cavity. ${ }^{10-14}$

There are reports of both endoscopic surgery ${ }^{15,16}$ and open suboccipital approaches ${ }^{17-20}$ for 4 th ventricular NCC, with good surgical and functional results. Minimally invasive endoscopic excision requires experience, particularly in cases with a thin aqueduct ${ }^{16,18,21,22}$ or if the cyst adheres to the ependymal lining. ${ }^{18,23}$ Reported complications include periventricular edema, residual cyst of the 4 th ventricle, need for reoperation, and intraoperative bleeding, ${ }^{6,17,24}$ although most reports did not describe serious complications. ${ }^{15,25}$

Indications for stereotactic neurosurgery are well defined. It remains the gold standard for locating deep lesions $<10 \mathrm{~mm}$, with extremely high localization precision (approximately $1 \mathrm{~mm}$ ). Difficulties of the procedure are mainly the need for intubation of the patient and incorrect positioning of the frame that may require repositioning. ${ }^{26}$ The procedure has been used to biopsy brainstem lesions via a suboccipital trepan with low complication rates. In 2014, a review of 20 publications including 457 patients who underwent stereotactic biopsy of lesions in the brainstem reported a rate of complications of $8.9 \% .{ }^{27}$ Another report of 78 patients found $<5 \%$ complications. ${ }^{28}$ Both studies concluded that stereotactic biopsy of deep intracranial lesions is an effective and safe procedure. ${ }^{28}$ There are no reports on stereotactic surgery for 4 th ventricular cysts.

On the basis of current knowledge of minimally invasive neurosurgery, and considering our extensive experience in stereotactic procedures, as well as our experience in neurosurgical management of patients with NCC, we considered that stereotactic resection of 4th ventricular cysts was a safe alternative for our patients. NCC cysts are not usually attached to the surrounding vascular nervous tissue, which facilitates their exeresis. ${ }^{2}$ In endoscopic and transcranial surgery, the cysts are extracted with traction or subtle aspiration, and in cases of subarachnoid NCC, the same principle plus irrigation with saline serum and intracranial pressure itself facilitate the excision of the cysts without major difficulties. When the cysts are free in the ventricular cavity, they can be extracted easily. In some cases, however, the cysts may be attached to surrounding vascular and nervous structures, which makes dissection more labor-intensive and riskier; the exact reason for this phenomenon is not known, and it is suggested that this occurs more frequently in patients treated with antiparasitic drugs before surgery.

During stereotactic surgery, if a cyst is attached to the ependyma, the cyst is ruptured, and most of the cyst membranes are very gently aspirated. Unlike hydatid cysts, rupture of NCC cysts is not associated with anaphylaxis or seeding of infection. To prevent injuries, traction is avoided. If any resistance is felt, the cyst membrane is fragmented using the cutter cannula mechanism, and aspiration continues until no more membranes are extracted and only CSF comes out. It is always possible that residual membrane fragments remain, but these should not result in clinical manifestations. In these cases, patient follow-up should confirm that the residual membranes do not cause any obstruction or inflammation.

The decision to use stereotactic resection as an additional, alternative surgical technique for these cases attempts to provide a less invasive approach, considering that neuroendoscopic access to the 4th ventricle is more difficult than accessing the lateral ventricles, and also considering that some patients with 4th ventricular NCC may require additional surgical procedures for other subarachnoid or large parenchymal lesions. Medical treatment is not recommended before cyst resection, because the destruction of the cysts is associated with neurological deterioration secondary to the inflammatory immune response to the death of the parasite. Although steroids such as prednisone or dexamethasone are commonly used to control this inflammatory process after degradation of viable cysts as an adjunct to cysticidal therapy, it seems preferable to delay antiparasitic regimes if surgical cyst extraction is planned. ${ }^{22,23,29}$

Neurocysticercosis continues to be a public health problem in endemic areas such as Peru; however, access to cysticidal treatment and the availability of minimally invasive neurosurgical techniques have improved its prognosis. In our center, stereotactic surgery is now used as an alternative in the neurosurgical treatment of patients with 4th ventricular NCC, and, although we have treated few cases to date, our experience has been satisfactory, and we will continue evaluating its performance.

\section{Lessons}

Stereotactic surgery offers an excellent minimally invasive alternative approach for 4th ventricular NCC. We applied our extensive knowledge and previous experience in the management of NCC and the availability 
of stereotaxis to safely treat these two patients with NCC cysts in the 4th ventricle.

\section{References}

1. Mahale RR, Mehta A, Rangasetty S. Extraparenchymal (racemose) neurocysticercosis and its multitude manifestations: a comprehensive review. J Clin Neurol. 2015;11(3):203-211.

2. Garcia $\mathrm{HH}$, Nash TE, Del Brutto $\mathrm{OH}$. Clinical symptoms, diagnosis, and treatment of neurocysticercosis. Lancet Neurol. 2014;13(12): 1202-1215.

3. Gripper LB, Welburn SC. Neurocysticercosis infection and disease-a review. Acta Trop. 2017;166:218-224.

4. Jensen TO, Post JJ. Intraventricular neurocysticercosis: presentation, diagnosis and management. Asian Pac J Trop Med. 2016;9(8):815-818.

5. Gupta P, Agrawal M, Sinha VD, Gupta A. Intraventricular racemose type neurocysticercosis with anterior interhemispheric fissure cyst: a rare case report. J Neurosci Rural Pract. 2015;6(2): 234-237.

6. Citow JS, Johnson JP, McBride DQ, Ammirati M. Imaging features and surgery-related outcomes in intraventricular neurocysticercosis. Neurosurg Focus. 2002;12(6):e6.

7. Peralta D, Banegas J, Miller E. Neurocisticercosisintraventricular tratada eficazmente con agentes anticestodos. Rev Med Hondur. 2012;80(3):108-110.

8. Del Brutto $\mathrm{OH}$. Neurocysticercosis: up-dating in diagnosis and treatment. Article in Spanish. Neurologia. 2005;20(8):412-418.

9. Sandoval-Balanzario MA, Rincón-Navarro RA, Granados-López R, Santos-Franco JA. Endoscopic third ventriculostomy for chronic communicating hydrocephalus in adults. Article in Spanish. Rev Med Inst Mex Seguro Soc. 2015;53(3):280-285.

10. Campbell BR, Reynoso D, White AC. Intraventricular neurocysticercosis and Bruns' syndrome: a review. J Rare Dis Res Treat. 2017;2(2):1-5.

11. Rana S, Prasad A, Brar R, et al. Caught in the act: migrating intraventricular neurocysticercosis causing intermittent unilateral hydrocephalus due to foramen of Monro obstruction. Acta Neurol Belg. 2018:118(3):509-511.

12. Araujo AL, Rodrigues RS, Marchiori E, et al. Migrating intraventricular cysticercosis: magnetic resonance imaging findings. Arq Neuropsiquiatr. 2008:66(1):111-113.

13. Thomas B, Krishnamoorthy T. Migrating intraventricular cysticercus during MRI. Neurology. 2005;65(8):1321.

14. García HH, Evans CAW, Nash TE, et al. Current consensus guidelines for treatment of neurocysticercosis. Clin Microbiol Rev. 2002;15(4):747-756.

15. Kaif M, Husain M, Ojha BK. Endoscopic management of intraventricular neurocysticercosis. Turk Neurosurg. 2019;29(1): $59-65$.

16. Goel RK, Ahmad FU, Vellimana AK, et al. Endoscopic management of intraventricular neurocysticercosis. J Clin Neurosci. 2008;15(10): 1096-1101.

17. Loyo M, Kleriga E, Estañol B. Fourth ventricular cysticercosis. Neurosurgery. 1980;7(5):456-458
18. Hanak BW, Walcott BP, Codd PJ, et al. Fourth ventricular neurocystercercosis presenting with acute hydrocephalus. J Clin Neurosci. 2011;18(6):867-869.

19. Franko LR, Pandian B, Gupta A, et al. Posterior fossa craniotomy for adherent fourth ventricle neurocysticercosis. Oper Neurosurg (Hagerstown). 2019;16(5):E154-E158.

20. Apuzzo ML, Dobkin WR, Zee CS, et al. Surgical considerations in treatment of intraventricular cysticercosis. An analysis of 45 cases. J Neurosurg. 1984;60(2):400-407.

21. Bergsneider M. Endoscopic removal of cysticercal cysts within the fourth ventricle. Technical note. J Neurosurg. 1999;91(2):340-345.

22. Coyle CM. Neurocysticercosis: an update. Curr Infect Dis Rep. 2014;16(11):437.

23. Rajshekhar V. Surgical management of neurocysticercosis. Int $\mathrm{J}$ Surg. 2010;8(2):100-104.

24. Nash TE, Ware JM, Mahanty S. Intraventricular neurocysticercosis: experience and long-term outcome from a tertiary referral center in the United States. Am J Trop Med Hyg. 2018;98(6):1755-1762.

25. Psarros TG, Zouros A, Coimbra C. Neurocysticercosis: a neurosurgical perspective. South Med J. 2003;96(10):1019-1022.

26. Fengqiang $L$, Jiadong $Q, Y i L$. Computer-assisted stereotactic neurosurgery with framework neurosurgery navigation. Clin Neurol Neurosurg. 2008;110(7):696-700.

27. Samadani U, Stein S, Moonis G, et al. Stereotactic biopsy of brain stem masses: Decision analysis and literature review. Surg Neurol. 2006;66(5):484-491.

28. Zorro Ó, Ordóñez Rubiano ÉG, Camacho JE, et al. Procedimientos neuroquirúrgicos cerebrales guiados por estereotaxia realizados en el Hospital Universitario de San Ignacio (HUSI): evolución a corto y mediano plazo. Univ Medica. 2012;54(1):39-52.

29. White AC Jr, Coyle CM, Rajshekhar V, et al. Diagnosis and Treatment of Neurocysticercosis: 2017 Clinical Practice Guidelines by the Infectious Diseases Society of America (IDSA) and the American Society of Tropical Medicine and Hygiene (ASTMH). Clin Infect Dis. 2018;66(8):e49-e75.

\section{Disclosures}

The authors report no conflict of interest concerning the materials or methods used in this study or the findings specified in this paper.

\section{Author Contributions}

Conception and design: Lines, Saavedra, Vásquez. Acquisition of data: Lines, Caucha, Félix. Analysis and interpretation of data: Lines, García, Medina. Drafting the article: Lines, García. Critically revising the article: Lines, García. Reviewed submitted version of manuscript: Lines, García, Antonio. Approved the final version of the manuscript on behalf of all authors: Lines. Administrative/technical/material support: Lines. Study supervision: Lines. Operating surgeon: Saavedra.

\section{Correspondence}

William W. Lines: Instituto Nacional De Ciencias Neurológicas, Lima Peru.williamincn@hotmail.com. 